\title{
Propiedades psicométricas de un cuestionario para evaluar expectativas académicas en estudiantes de primer año de Medicina
}

\author{
CRISTHIAN PÉREZ V. , $^{1, \mathrm{a}}$, LILIANA ORTIZ M.1,b, \\ EDUARDO FASCE H. ${ }^{1}$, PAULA PARRA P. ${ }^{1, b, c}$, OLGA MATUS B..$^{1, b, d}$, \\ PETER MCCOLL C. ${ }^{2, b}$, GRACIELA TORRES A. ${ }^{3, b, e}$, ANDREA MEYER K. ${ }^{4, b}$, \\ CAROLINA MÁRQUEZ U., ${ }^{1, b, f}$, JAVIERA ORTEGA B. ${ }^{1, g}$
}

\section{Assessment of psychometric properties of the academic involvement questionnaire, expectations version}

Background: Academic Involvement Questionnaire, Expectations version (CIA-A), assesses the expectations of involvement in studies. It is a relevant predictor of student success. However, the evidence of its validity and reliability in Chile is low, and in the case of Medical students, there is no evidence at all. Aim: To evaluate the factorial structure and internal consistency of the CIA-A in Chilean Medical school freshmen. Material and Methods: The survey was applied to 340 Medicine freshmen, chosen by non-probability quota sampling. They answered a back-translated version of CIA-A from Portuguese to Spanish, plus a sociodemographic questionnaire. For psychometric analysis of the CIA-A, an exploratory factor analysis was carried on, the reliability of the factors was calculated, a descriptive analysis was conducted and their correlation was assessed. Results: Five factors were identified: vocational, institutional and social involvement, use of resources and student participation. Their reliabilities ranged between Cronbach's alpha values of 0.71 to 0.87 . Factors also showed statistically significant correlations between each other. Conclusions: Identified factor structure is theoretically consistent with the structure of original version. It just disagrees in one factor. In addition, the factors' internal consistency were adequate for using them in research. This supports the construct validity and reliability of the CIA-A to assess involvement expectations in medical school freshmen.

(Rev Med Chile 2015; 143: 1459-1467)

Key words: Learning; Medical Education, Undergraduate; Students, $\mathrm{Me-}$ dical.

$\tau$ a creciente preocupación por la calidad de los centros formativos universitarios presiona por mejorar los aprendizajes que adquieren sus estudiantes y su pertinencia ante las necesidades sociales ${ }^{1,2}$, lo que en el caso de Medicina implica adaptarse a cambios demográficos y sanitarios, enfrentar exitosamente la rápida
'Departamento de Educación

Médica, Universidad de

Concepción, Chile.

${ }^{2}$ Escuela de Medicina sede Viña del Mar, Facultad de Medicina, Universidad Andres Bello, Chile.

${ }^{3}$ Facultad de Medicina,

Universidad San Sebastián, Chile.

${ }^{4}$ Facultad de Medicina,

Universidad Católica de la

Santísima Concepción, Chile.

aPsicólogo, Magíster en Psicología

con mención en Psicología

Educativa.

'Magíster en Educación Médica

para Ciencias de la Salud.

cKinesióloga.

¿Ingeniero Informático.

eEnfermera matrona.

'Bioquímica.

gPsicóloga, Magíster en Ciencias

de la Educación.

Trabajo financiado por el proyecto FONDECYT Regular 1121002

Recibido el 7 de mayo de 2015 aceptado el 30 de julio de 2015

Correspondencia a:

Cristhian Pérez Villalobos Departamento de Educación Médica, Facultad de Medicina, Universidad de Concepción Víctor Lamas 1290, Concepción, Chile.

Teléfono: +56 412204932 cperezv@udec.cl actualización de los conocimientos disciplinares y asumir un rol social desde la universidad ${ }^{3}$. Sin embargo, también implica mejorar la calidad de vida universitaria ${ }^{1}$ y los niveles de bienestar del alumnado ${ }^{4,5}$.

Ante estas nuevas exigencias, ha crecido el interés por investigar e intervenir distintos aspectos 
de la formación de carreras como Medicina, tales como su currículo ${ }^{6}$, sus procesos de enseñanza, aprendizaje y evaluación ${ }^{1,3}$, las competencias de los docentes ${ }^{7-10}$ y el ambiente formativo ${ }^{11-14}$.

Lo anterior se entiende desde una perspectiva contextual del éxito académico. Pero ésta es complementaria a una perspectiva del desarrollo, preocupada por los aspectos intrapsíquicos del estudiantado que afectan su adaptación universi$\operatorname{taria}^{15}$. Estos factores individuales son relevantes ante los desafíos adaptativos que enfrenta todo alumno terciario: una estructura formativa más flexible y menos secuenciada, nuevos compañeros, cambios en la forma de vida, etc. Dichos cambios requieren autonomía, madurez y habilidades sociales del estudiante para adaptarse a la nueva etapa $^{16}$. En el caso de Medicina, caracterizada por altos niveles de estrés ${ }^{17}$, demanda características personales complejas que permitan al alumnado alcanzar el éxito y gestionarse adecuadamente durante su formación ${ }^{18}$.

Hay múltiples estudios preocupados por abordar aspectos intrapsíquicos de los estudiantes de Medicina, pero son pocas las investigaciones que han abordado sus expectativas académicas ${ }^{19}$. Esto, aunque serían claves para entender la forma en que los individuos se adaptan a la universidad, al influir en su afrontamiento de la carrera, su adaptación, persistencia y desempeño académico ${ }^{16,19,20}$.

Las expectativas académicas se entienden como las creencias, motivaciones y afectos que los alumnos asocian a la educación superior ${ }^{16}$. No obstante, es posible evidenciar en la literatura una confusión entre expectativas (lo que se cree que va a suceder) y aspiraciones (lo que se desea que suceda $)^{21}$.

En esta línea, estudios en estudiantes de Medicina sobre sus expectativas del perfil de egreso ${ }^{22}$, de los profesores ${ }^{23} \mathrm{y}$ de la formación ${ }^{19}$ se dirigen más a evaluar aspiraciones que expectativas. Las aspiraciones son importante por su efecto potencial en el tipo de motivación que orienta la actividad de los alumnos ${ }^{24}$.

Sin embargo, también son importantes las expectativas, como tales, pues una visión realista de lo que ocurre después de la vida universitaria (empleabilidad, remuneraciones, calidad de vida laboral, etc.) puede evitar la deserción ${ }^{25}$.

Entre estas expectativas destacan aquellas de implicación, o qué espera hacer el estudiante durante su vida universitaria, que se asociarían al éxito académico que éste logra, a su satisfacción académica y a su implicación conductual en el proceso formativo ${ }^{1,15}$. Por ello, evaluar las expectativas de implicación constituiría un buen screening para diagnosticar a los alumnos y anticipar problemas de adaptación ${ }^{15}$.

En Chile, hay un estudio sobre expectativas de implicación en estudiantes universitarios de diversas áreas del conocimiento ${ }^{26}$. En éste, se empleó el Cuestionario de Implicación Académica, forma A o versión Expectativas (CIA-A), diseñado $y$ validado en estudiantes portugueses por Soares y Almeida ${ }^{1}$. Este estudio aportó evidencia de la confiabilidad y validez de contenido (mediante jueces expertos) del CIA-A en Chile. No obstante, reconoció la necesidad de analizar otras propiedades psicométricas y no incluyó en su muestra a estudiantes de la salud ${ }^{26}$.

En vista de lo anterior, y ante la utilidad potencial de contar con un instrumento para medir expectativas de implicación en estudiantes de Medicina, el presente estudio evalúa el CIA-A en esta población, analizando la estructura factorial del cuestionario, como evidencia de su validez de constructo y su consistencia interna, como evidencia de la precisión de sus puntuaciones ${ }^{27}$.

\section{Método}

\section{Participantes}

Mediante muestreo no probabilístico por cuotas se accedió a 340 estudiantes de primer año, provenientes de cinco escuelas de Medicina de Chile. Se incluyó tres regiones del país. De éstos, se eliminó a quienes tenían respuestas omitidas en el cuestionario analizando, quedando una muestra válida de 320 estudiantes (Tabla 1).

\section{Instrumento}

Los estudiantes respondieron una retrotraducción del portugués al castellano del Cuestionario de Implicación Académica de Soares y Almeida ${ }^{1}$, realizada para este estudio. Específicamente, la forma A (CIA-A) que evalúa expectativas. La forma $B$, que evalúa comportamientos de implicación, no fue considerada esta vez.

Este cuestionario de auto-respuesta está constituido por 38 afirmaciones sobre situaciones que un estudiante puede enfrentar en su vida universitaria $\mathrm{y}$ ante las cuales debe responder según el grado 
Tabla 1. Caracterización de la muestra de los estudiantes de primer año de Medicina

\begin{tabular}{|lll|}
\hline Variable & Estadísticos descriptivos & \\
\hline Edad & M =19,34; DE $=2,04 ;$ Mín $=18 ;$ & Máx $=33$ \\
\hline Sexo & Hombre & $(\mathrm{n}=188 ; 58,75 \%)$ \\
& Mujer & $(\mathrm{n}=130 ; 40,62 \%)$ \\
& Omisiones & $(\mathrm{n}=2 ; 0,62 \%)$ \\
\hline Establecimiento de origen & Municipalizado & $(\mathrm{n}=42 ; 13,12 \%)$ \\
& Particular subvencionado & $(\mathrm{n}=138 ; 43,12 \%)$ \\
& Particular pagado & $(\mathrm{n}=139 ; 43,44 \%)$ \\
& Omisiones & $(\mathrm{n}=1 ; 0,31 \%)$ \\
Universidad & Pertenecientes al CRUCH & $(\mathrm{n}=155 ; 48,44 \%)$ \\
& No pertenecientes al CRUCH & $(\mathrm{n}=165 ; 51,56 \%)$ \\
Región & Región de Valparaíso & $(\mathrm{n}=23 ; 7,19 \%)$ \\
& Región Metropolitana & $(\mathrm{n}=65 ; 20,31 \%)$ \\
& Región del Bío Bío & $(\mathrm{n}=232 ; 72,50 \%)$ \\
Carrera previa & No & $(\mathrm{n}=240 ; 75,00 \%)$ \\
& Sí, pero no la terminó & $(\mathrm{n}=61 ; 19,06 \%)$ \\
& Sí, y la terminó & $(\mathrm{n}=12 ; 3,75 \%)$ \\
& Omisiones & $(\mathrm{n}=7 ; 2,19 \%)$ \\
\hline
\end{tabular}

$\mathrm{N}=320 ; \mathrm{M}=$ Media aritmética; $\mathrm{DE}=$ Desviación estándar; Mín = Mínimo; Máx = Máximo; $\mathrm{n}$ = Frecuencia absoluta.

en que espera que sucedan, utilizando una escala tipo Likert de cinco alternativas $(1=$ nunca o casi nunca; 2 = rara vez; 3 = a veces; $4=$ frecuentemente; $5=$ siempre o casi siempre). De acuerdo a sus autores mide cinco áreas: Implicación institucional, implicación vocacional, implicación social, utilización de recursos e implicación curricular ${ }^{1}$.

Adicionalmente, se aplicó un cuestionario sociodemográfico para describir la muestra.

\section{Procedimiento}

El cuestionario fue aplicado por encuestadores capacitados a los estudiantes antes de terminar el primer mes de clases del primer año. La aplicación se realizó en instalaciones de sus universidades, previo proceso de consentimiento informado. El procedimiento fue evaluado y aprobado por la Comisión Nacional de Investigación Científica y Tecnológica de Chile, CONICYT.

\section{Análisis de datos}

Implicó dos procedimientos: Primero, se realizó un análisis factorial exploratorio (AFE) para determinar la dimensionalidad interna del cuestionario, a fin de aportar evidencia de su validez de constructo. El AFE empleó el método de extracción del análisis de ejes principales (AEP), recomendado para este tipo de instrumentos ${ }^{27,28}$.

Posteriormente, se evaluó la consistencia interna de los factores identificados con el coeficiente de confiabilidad alfa de Cronbach. Finalmente, se realizó un análisis descriptivo de los factores y se evaluó la correlación entre éstos aplicando el coeficiente de Pearson. Se consideró un valor de $\mathrm{p}<0,05$ como estadísticamente significativo. Para analizar los datos se usó STATA SE 11.0.

\section{Resultados}

Como primer paso del AFE, se evaluó su pertinencia. Considerando los 38 ítems, se calculó el estadístico de adecuación muestral de Kaiser-Meyer-Olkin (KMO), que fue de 0,91 , y la prueba de esfericidad de Barlett, que fue estadísticamente significativa, $\chi^{2}(703)=5039,21 ; p<0,001$. Ambos resultados respaldaron la pertinencia del análisis.

El siguiente paso implicó estimar el número de factores, empleando dos criterios: Kaiser-Guttman o de raíz latente ${ }^{30} \mathrm{y}$ análisis paralelo de Horn ${ }^{27}$. El primer criterio indicó la existencia de cinco factores con valores propios (eigenvalues) mayores a 1,0 $(10,34,1,60,1,41,1,33$ y 1,19$)$, explicando $89,66 \%$ de la varianza total de los ítems. El análisis paralelo, 
en base a 1.000 muestras aleatorias, coincidió con la existencia de cinco factores con valores propios $(10,34 ; 1,60 ; 1,41 ; 1,33$ y 1,19$)$ por sobre los valores propios presentados por $95 \%$ de las muestras aleatorias $(0,85 ; 0,73 ; 0,68 ; 0,65$ y 0,62$)$.

Ante el acuerdo entre factores, el tercer paso fue calcular la distribución de los ítems en una estructura de cinco factores, mediante AEP con rotación oblicua Promax. Sin embargo, cuatro de los 38 ítems (ítems 1, 6, 15 y 16) presentaron coeficientes bajo 0,30 , el valor mínimo para considerar una carga factorial como estadísticamente significativa ${ }^{30}$. Por ello, se decidió eliminar al ítem 6 ("Acceder a información relacionada con mis asignaturas a través de las redes de Internet de mi universidad"), que presentaba los valores más bajos, y repetir el análisis completo con los ítems restantes. Mediante el mismo procedimiento, se debió eliminar el ítem 15 ("Asistir con constancia y puntualidad a clases") y luego el 16 ("Reunirme con estudiantes de las diferentes carreras de la universidad para divertirme o conversar").

Finalmente, para los 35 ítems remanentes, el estadístico KMO fue de 0,91, y la prueba de Barlett, estadísticamente significativa, $\chi^{2}(595)=$ 4691,12; p < 0,001, apoyando la realización del AFE. Al estimar el número de factores, el criterio de Kaiser-Guttman indicó cinco factores con valores propios (eigenvalues) mayores a 1,0 $(9,92$, $1,46,1,33,1,30$ y 1,16 ), explicando $93,07 \%$ de la varianza total de los ítems. El análisis paralelo, en base a 1.000 muestras aleatorias, también estimó cinco factores con valores propios $(9,92 ; 1,46$; $1,33 ; 1,30$ y 1,16$)$ por sobre los valores propios presentados por $95 \%$ de las muestras aleatorias $(0,84 ; 0,68 ; 0,60 ; 0,54$ y 0,52$)$.

Al calcular los coeficientes de configuración para los cinco factores, utilizando AEP con rotación oblicua Promax, Tabla 2, se encontró que todos los ítems presentaban cargas sobre 0,30 , aunque uno de ellos (ítem 10) presentó cargas sobre dicho umbral en dos factores (carga cruzada). Se asignaron los ítems a los factores en donde presentaban cargas sobre este umbral y el ítem 10 fue asignado al ítem en el que presentaba mayor pertinencia conceptual.

Tabla 2. Matriz de configuración del Cuestionario de Implicación Académica, versión Expectativas en alumnos de primer año de Medicina obtenida mediante análisis de eje principal con rotación Promax

\begin{tabular}{|c|c|c|c|c|c|}
\hline Ítem & $\mathbf{I}$ & II & III & IV & $\mathbf{v}$ \\
\hline 1. Participar activamente en las clases & $0,305^{a}$ & 0,045 & 0,090 & 0,070 & 0,045 \\
\hline $\begin{array}{l}\text { 2. Usar o manipular tecnologías avanzadas disponibles en la universidad } \\
\text { (equipos informáticos, electrónicos, de laboratorio, etc.) }\end{array}$ & 0,317 & $-0,094$ & 0,064 & 0,205 & 0,085 \\
\hline $\begin{array}{l}\text { 3. Evaluar si las materias de las asignaturas que curso realmente } \\
\text { corresponden a mis intereses académicos o profesionales }\end{array}$ & 0,344 & $-0,043$ & 0,253 & $-0,034$ & $-0,042$ \\
\hline 4. Leer la bibliografía recomendada antes de cada clase & 0,202 & 0,338 & 0,039 & 0,006 & $-0,060$ \\
\hline $\begin{array}{l}\text { 5. Utilizar los espacios recreativos disponibles en la universidad (cafeterías, } \\
\text { gimnasios, recintos deportivos, etc.) }\end{array}$ & $-0,039$ & $-0,037$ & 0,115 & 0,349 & 0,148 \\
\hline $\begin{array}{l}\text { 7. Participar activamente en los grupos u organizaciones de estudiantes de } \\
\text { mi universidad (centros de alumnos, asociaciones de estudiantes, etc.) }\end{array}$ & $-0,018$ & $-0,085$ & 0,187 & 0,003 & 0,736 \\
\hline $\begin{array}{l}\text { 8. Obtener información sobre las profesiones o empleos más relacionados } \\
\text { con mi carrera }\end{array}$ & 0,525 & $-0,049$ & 0,118 & $-0,027$ & 0,120 \\
\hline $\begin{array}{l}\text { 9. Hablar con mis compañeros de la universidad sobre asuntos de } \\
\text { actualidad }\end{array}$ & 0,117 & $-0,168$ & 0,573 & 0,032 & 0,189 \\
\hline $\begin{array}{l}\text { 10. Trabajar con mis profesores en los proyectos de investigación que estén } \\
\text { desarrollando }\end{array}$ & 0,463 & 0,184 & $-0,102$ & $-0,150$ & 0,390 \\
\hline $\begin{array}{l}\text { 11. Usar los espacios de la universidad para hacer los trabajos individuales y } \\
\text { grupales }\end{array}$ & $-0,096$ & $-0,011$ & 0,176 & 0,572 & 0,076 \\
\hline 12. Tomar notas o apuntes durante las clases & $-0,060$ & 0,086 & 0,089 & 0,459 & $-0,164$ \\
\hline
\end{tabular}




\begin{tabular}{|c|c|c|c|c|c|}
\hline 13. Usar la biblioteca como un espacio para leer, estudiar o investigar & 0,137 & $-0,179$ & 0,143 & 0,590 & $-0,124$ \\
\hline $\begin{array}{l}\text { 14. Conversar con mis profesores sobre mis planes e intereses educativos } \\
\text { y/o profesionales }\end{array}$ & 0,191 & 0,415 & 0,129 & $-0,028$ & 0,215 \\
\hline 17. Realizar actividades prácticas asociadas a mi carrera para ver si me gustan & 0,818 & $-0,106$ & $-0,091$ & $-0,081$ & 0,234 \\
\hline $\begin{array}{l}\text { 18. Utilizar los computadores disponibles en la universidad para hacer } \\
\text { trabajos e informes }\end{array}$ & 0,069 & 0,074 & $-0,289$ & 0,562 & 0,257 \\
\hline $\begin{array}{l}\text { 19. Asumir funciones en cargos académicos de la universidad (delegado } \\
\text { de curso, representante de los alumnos ante la Facultad, etc.) }\end{array}$ & $-0,068$ & 0,049 & $-0,038$ & 0,112 & 0,701 \\
\hline $\begin{array}{l}\text { 20. Hablar con otros estudiantes sobre asuntos relacionados con las } \\
\text { asignaturas que curso }\end{array}$ & 0,031 & $-0,043$ & 0,573 & 0,108 & 0,025 \\
\hline $\begin{array}{l}\text { 21. Aplicar los conocimientos aprendidos en mis asignaturas en otras } \\
\text { áreas de la vida (en el trabajo, en las relaciones con amigos, familiares } \\
\text { y colegas, etc.) }\end{array}$ & 0,611 & $-0,146$ & 0,154 & 0,117 & 0,007 \\
\hline $\begin{array}{l}\text { 22. Establecer relaciones más cercanas con algunos de mis compañeros } \\
\text { de la universidad }\end{array}$ & 0,119 & $-0,068$ & 0,429 & 0,180 & 0,050 \\
\hline 23. Consultar un especialista en orientación vocacional en la universidad & $-0,097$ & 0,547 & $-0,066$ & 0,044 & 0,217 \\
\hline $\begin{array}{l}\text { 24. Tener profesores que expliquen la utilidad prácticas de las materias } \\
\text { abordadas }\end{array}$ & 0,564 & 0,042 & 0,023 & 0,141 & $-0,211$ \\
\hline $\begin{array}{l}\text { 25. Utilizar los computadores de la universidad para consultar los materiales } \\
\text { de su biblioteca y de otras }\end{array}$ & $-0,024$ & 0,239 & $-0,188$ & 0,605 & 0,113 \\
\hline $\begin{array}{l}\text { 26. Conversar con personas especializadas en el área profesional de mi } \\
\text { carrera }\end{array}$ & 0,567 & 0,104 & 0,084 & 0,096 & 0,001 \\
\hline $\begin{array}{l}\text { 27. Ir a reuniones convocadas por grupos de estudiantes de la universidad } \\
\text { (centros de alumnos, asociaciones de estudiantes, etc.) }\end{array}$ & 0,009 & 0,082 & 0,258 & $-0,099$ & 0,497 \\
\hline $\begin{array}{l}\text { 28. Tener conversaciones serias con estudiantes cuyas creencias y valores sean } \\
\text { diferentes a los míos }\end{array}$ & $-0,115$ & 0,168 & 0,718 & $-0,019$ & 0,045 \\
\hline $\begin{array}{l}\text { 29. Estar atento a la evolución del mercado laboral en el área profesional } \\
\text { de mi carrera }\end{array}$ & 0,492 & 0,233 & 0,061 & 0,030 & $-0,106$ \\
\hline $\begin{array}{l}\text { 30. Hablar con mis profesores después de las clases sobre temas relacionados } \\
\text { con sus disciplinas }\end{array}$ & 0,254 & 0,526 & 0,139 & $-0,085$ & $-0,063$ \\
\hline 31. Desarrollar las competencias profesionales relacionadas con mi carrera & 0,816 & 0,051 & $-0,112$ & $-0,071$ & $-0,108$ \\
\hline 32. Hablar con mis profesores sobre mis dificultades y problemas personales & $-0,180$ & 0,775 & 0,075 & 0,045 & 0,046 \\
\hline $\begin{array}{l}\text { 33. Hacer lecturas adicionales para realizar los trabajos de mis asignaturas y } \\
\text { preparar los exámenes }\end{array}$ & 0,229 & 0,335 & $-0,005$ & 0,159 & $-0,250$ \\
\hline $\begin{array}{l}\text { 34. Aprovechar mis conocimientos y experiencia personal en las discusiones } \\
\text { en clases y/o para la realización de trabajos o exámenes }\end{array}$ & 0,208 & 0,081 & 0,411 & 0,166 & $-0,098$ \\
\hline $\begin{array}{l}\text { 35. Utilizar los servicios de apoyo social y psicológico ofrecidos por la } \\
\text { universidad }\end{array}$ & $-0,019$ & 0,594 & $-0,008$ & 0,136 & 0,095 \\
\hline $\begin{array}{l}\text { 36. Pedir a los profesores que me aconsejen para mejorar mi rendimiento } \\
\text { académico }\end{array}$ & 0,100 & 0,750 & 0,013 & $-0,098$ & $-0,103$ \\
\hline 37. Hablar con otros estudiantes sobre temas sociales y éticos & $-0,097$ & 0,186 & 0,701 & $-0,107$ & 0,051 \\
\hline $\begin{array}{l}\text { 38. Llevar a cabo actividades prácticas relacionadas con mi carrera para } \\
\text { evaluar mis capacidades y/o desempeño }\end{array}$ & 0,842 & 0,031 & $-0,094$ & $-0,086$ & 0,032 \\
\hline Autovalores & 9,92 & 1,46 & 1,33 & 1,30 & 1,16 \\
\hline$\%$ de varianza total & 60,81 & 8,98 & 8,16 & 7,99 & 7,13 \\
\hline
\end{tabular}

aLas cursivas indican cargas superiores a 0,30. 
Tabla 3. Estadísticos descriptivos de los factores del Cuestionario de Implicación Académico, versión Expectativas en estudiantes de primer año de Medicina

\begin{tabular}{|c|c|c|c|c|c|}
\hline Estadísticos & $\begin{array}{l}\text { Implicación } \\
\text { vocacional }\end{array}$ & $\begin{array}{l}\text { Implicación } \\
\text { institucional }\end{array}$ & $\begin{array}{l}\text { Implicación } \\
\text { social }\end{array}$ & $\begin{array}{l}\text { Utilización de } \\
\text { recursos }\end{array}$ & $\begin{array}{c}\text { Participación } \\
\text { estudiantil }\end{array}$ \\
\hline Media aritmética & 49,29 & 25,06 & 24,39 & 24,07 & 8,04 \\
\hline Desviación estándar & 7,18 & 5,92 & 3,74 & 3,70 & 2,67 \\
\hline Percentil 25 & 45,00 & 21,00 & 22,00 & 22,00 & 6,00 \\
\hline Percentil 50 & 50,00 & 25,00 & 25,00 & 24,00 & 8,00 \\
\hline Percentil 75 & 55,00 & 29,00 & 27,00 & 27,00 & 10,00 \\
\hline Asimetría & $-0,95$ & $-0,02$ & $-0,63$ & $-0,67$ & 0,19 \\
\hline Curtosis & 4,04 & 2,55 & 3,23 & 4,31 & 2,81 \\
\hline \multicolumn{6}{|c|}{ Niveles de interpretación } \\
\hline Bajo & $\leq 44$ & $\leq 20$ & $\leq 21$ & $\leq 21$ & $\leq 5$ \\
\hline Medio & $45-54$ & $21-28$ & $22-26$ & $22-26$ & $6-9$ \\
\hline Alto & $\geq 55$ & $\geq 29$ & $\geq 27$ & $\geq 27$ & $\geq 10$ \\
\hline
\end{tabular}

$N=320$.

Con esta asignación, los 35 ítems restantes del cuestionario quedaron organizados como sigue:

- Factor I: Incluyendo los ítems 38, 17, 31, 21, $26,24,8,29,10,3,2$ y 1 (ordenados de mayor a menor carga), se denominó Implicación vocacional. Su alfa de Cronbach fue de $a=$ 0,87 , con correlaciones entre los ítems y el total corregido desde $r=0,41$ (ítem 1) a $r=$ 0,71 (ítem 17).

- Factor II: Incluyendo los ítems 32, 36, 35, 23, 30, 14, 4 y 33, se denominó Implicación institucional, y mostró un alfa de Cronbach de $\alpha=$ 0,84 , con correlaciones ítems-total corregido desde $r=0,40$ (ítem 33) a $r=0,66$ (ítem 36).

- Factor III: Considerando los ítems 28, 37, 9, 20, 22 y 34, se etiquetó como Implicación social y presentó un $\alpha=0,81$. Sus correlaciones ítems-total corregido fueron desde $r=0,48$ (ítem 22) a $r=0,66$ (ítem 28).

- Factor IV: Reuniendo los ítems 25, 13, 11, 18, 12 y 5 , se llamó Utilización de recursos, con un $\alpha=0,71$ y con correlaciones ítems-total corregido desde $r=0,31$ (ítem 12) a $r=0,56$ (ítem 25).

- Factor V: Subsumiendo los ítems 7, 19 y 27, se denominó Participación estudiantil. Su confiabilidad fue de $\alpha=0,77$, con correlaciones ítems-total corregido desde $r=0,54$ (ítem 27) a $r=0,65$ (ítem 7).
A partir de esto, se procedió a calcular los puntajes de cada factor mediante la sumatoria de los ítems y se realizó un análisis descriptivo de los mismos, a fin de tener valores de referencia para futuras utilizaciones del CIA-A (Tabla 3). En dicha tabla se sugiere que, con fines heurísticos, un puntaje inferior al percentil 25 en cualquier factor sea considerado como "Nivel bajo", un valor igual o mayor al percentil 25 y bajo el percentil 75 como "Nivel medio", y un puntaje igual o mayor al percentil 75 como "Nivel alto".

Finalmente, se evaluó la correlación de los factores identificados. Los resultados mostraron correlaciones directas estadísticamente significativas entre todos los factores (Tabla 4).

\section{Discusión}

Las expectativas académicas son un aspecto importante a considerar en la formación de estudiantes de Medicina, pues afectan cómo ellos interactúan con su currículo ${ }^{19}$. Lo anterior se vuelve preocupante ante la evidencia que muestra que las expectativas de los estudiantes sobre el grado de interacción social, aprendizaje e implicación académica que lograrán suelen ser irreales, pudiendo generarles dificultades en sus estudios, e incluso favorecer el abandono y el fracaso académico ${ }^{31}$. 
Tabla 4. Correlaciones entre los factores del Cuestionario de Implicación Académico, versión Expectativas en estudiantes de primer año de Medicina

\begin{tabular}{|c|c|c|c|c|c|}
\hline & 1 & 2 & 3 & 4 & 5 \\
\hline 1. Implicación vocacional & $0,87^{a}$ & & & & \\
\hline 2. Implicación institucional & $0,65^{* * *}$ & $0,84^{a}$ & & & \\
\hline 3. Implicación social & $0,58^{* * *}$ & $0,51 * * *$ & $0,81^{\text {a }}$ & & \\
\hline 4. Utilización de recursos & $0,47^{* * *}$ & $0,43^{* * *}$ & $0,38^{* * *}$ & $0,71^{\mathrm{a}}$ & \\
\hline 5. Participación estudiantil & $0,46^{* * *}$ & $0,44^{* * *}$ & $0,43^{* * *}$ & $0,33^{* * *}$ & $0,77^{\mathrm{a}}$ \\
\hline
\end{tabular}

$\mathrm{N}=320 ;{ }^{*}: \mathrm{p}<0,05 ;{ }^{* *}: \mathrm{p}<0,01 ;{ }^{* * *}: \mathrm{p}<0,001$. ${ }^{a}$ Coeficiente Alfa de Cronbach.

De esta manera, contar con una herramienta de tamizaje de las expectativas de implicación académica, como el CIA-A, puede permitir diagnósticos tempranos e identificar, oportunamente, perfiles de estudiantes que podrían enfrentar problemas de adaptación universitaria ${ }^{15}$.

El presente estudio aporta evidencia de validez de constructo y confiabilidad de este cuestionario en estudiantes de Medicina. Esto, atendiendo a las sugerencias de Abello ${ }^{26}$ de contar con más evidencias psicométricas de éste en Chile. Además, incluye una población estudiantil caracterizada por un alto nivel de exigencia ${ }^{17}$.

Para ello, a partir del AFE, se respalda una estructura de cinco factores:

1. Implicación vocacional, que apunta a la búsqueda de actividades que favorezcan el logro de los objetivos y planes de carrera ${ }^{16}$.

2. Implicación institucional, que refiere al acceso activo del alumno a recursos y apoyos universitarios ${ }^{16}$, como planta docente, otros profesionales y bibliografía para fortalecer su desarrollo académico y personal.

3. Implicación social, referido al grado en que el estudiante espera relacionarse con sus compañeros universitarios, establecer vínculos cercanos, compartir experiencias académicas y participar en instancias de reflexión en temas relevantes para la comunidad.

4. Utilización de recursos, que alude al acceso y uso de espacios y recursos bibliográficos, tecnológicos y recreativos durante la formación universitaria.

5. Participación estudiantil, que refiere al grado de participación que los estudiantes esperan tener en asociaciones estudiantiles y en actividades organizadas por este estamento.
Los primeros cuatro factores muestran una adecuada coherencia conceptual con la propuesta del instrumento original ${ }^{1,16}$, coincidiendo además en la mayoría de los ítems con los factores homónimos de la versión portuguesa.

Las discrepancias surgen en el factor Participación estudiantil, que no aparece en la versión original $^{1}$ y se constituye por los ítems que en el factor Implicación institucional, de dicha versión, aludían a la participación en actividades representativas intra-universitarias. Los resultados muestran que los estudiantes de Medicina diferencian la participación referida directamente a sus propios procesos de aprendizaje (Implicación institucional), de aquellos más vinculados a participación política, evidenciando preocupantemente una separación de los procesos académicos y la participación.

Por otro lado, el factor Implicación curricular de la versión original ${ }^{1}$ desaparece en este estudio y sus ítems se desagregan en los factores de Implicación vocacional e institucional, y en Uso de Recursos. Este factor había mostrado la menor confiabilidad en la versión original ${ }^{16}$, y su desaparición se debería a que, al referirse a actividades académicas generales, habría sido desagregado por la población chilena según el área en que ocurrían.

Lo anterior muestra que la estructura factorial identificada sería teóricamente plausible, y apoyaría el uso de los cinco puntajes identificados para evaluar expectativas de implicación académica. Complementario a esto, el análisis de confiabilidad mostró que los tres primeros factores tienen buena consistencia interna y los dos últimos son aceptables $^{32}$. Así, su precisión es adecuada para ser usada en diagnósticos e investigación, aunque no suficiente para la toma de decisiones (p.e. selección de estudiantes, promoción, etc. $)^{27,29}$. 
A partir de este respaldo, se calculó y realizó un análisis descriptivo de los factores del CIA-A, proponiendo una clasificación de los puntajes de los sujetos basados en los percentiles 25 y 75 . Sin embargo, aunque el estudio cubre tres regiones y diversas escuelas de Medicina, se presentan estos baremos sólo como un marco de referencia, dejando como una línea de investigación futura la construcción de baremos en una muestra más amplia y heterogénea.

Finalmente, los factores muestran correlaciones directas entre sí, esperables dado que las expectativas académicas, al abordar el mismo fenómeno, deberían ser interdependientes. Por otro lado, la intensidad de las correlaciones no fue alta, evidenciando que el cuestionario evalúa cinco aspectos diferenciados de las expectativas académicas.

En conclusión, se presenta evidencia a favor de la validez y confiabilidad del CIA-A para ser usado en la evaluación de expectativas de implicación académica en estudiantes de Medicina de Chile, diferenciando lo que se espera lograr en términos de trabajo académico, búsqueda de apoyo institucional, uso de los recursos disponibles, relaciones interpersonales y participación estudiantil, aspectos que son relevantes tanto para el diagnóstico educativo como para la definición de áreas prioritarias para la intervención intrauniversitaria.

Sin embargo, queda pendiente para estudios posteriores, además de la construcción de baremos, la evaluación de la validez de criterio del cuestionario y de su estabilidad temporal.

\section{Referencias}

1. Almeida LS, Gonçalves A, Salgueira AP, Soares AP, Machado C, Fernandes EM, et al. Expectativas de envolvimento académico à entrada na universidade: estudo com alunos da Universidade do Minho. Psicologia: Teoria, Investigação e Prática 2003; 1: 3-15.

2. Jouannet C, Salas MH, Contreras MA. Modelo de implementación de Aprendizaje Servicio (A+S) en la UC: Una experiencia que impacta positivamente en la formación profesional integral. Calidad en la Educación 2013; 39: 197-212.

3. Castillo M, Hawes G, Castillo S, Romero L, Rojas AM, Espinoza M, et al. Cambio educativo en las Facultades de Medicina. Rev Med Chile 2014; 142 (8): 1056-60.

4. Pérez C, Bonnefoy C, Cabrera A, Peine S, Macaya K,
Baqueano M, et al. Análisis, desde la psicología positiva, de la salud mental en alumnos universitarios de primer año de Concepción, Chile. Av Psicol Latinoam 2011; 29 (1): 148-60.

5. Pérez C, Bonnefoy C, Cabrera A, Peine S, Macaya K, Baqueano $\mathrm{M}$, et al. Problemas de salud mental en alumnos universitarios de primer año de Concepción, Chile. An Psicol 2012; 28 (3): 797-804.

6. Ortiz L, Gajardo J. Propuesta de competencias genéricas para el perfil de egreso del médico cirujano de la Universidad de Concepción, Chile. Educ Med Super 2014; 28 (3): 424-35.

7. Triviño X, Sirhan M, Moore P, Montero L. Impacto de un programa de formación en docencia en una escuela de medicina. Rev Med Chile 2011; 139: 1508-15.

8. Montero L, Triviño X, Sirhan M, Moore P, Leiva L. Barreras para la formación en docencia de los profesores de medicina: una aproximación cualitativa. Rev Med Chile 2012; 140: 695-702.

9. Sirhan M, Triviño X. Evaluación de una experiencia de capacitación en planificación educacional para directores de programas de las especialidades médicas. Rev Med Chile 2012; 140: 530-7.

10. Pérez C, Fasce E, Coloma K, Vaccarezza G, Ortega J. Percepción de académicos de carreras de la salud de Chile sobre el perfeccionamiento docente. Rev Med Chile 2013; 141: 787-92.

11. Al-hazimi A, Al-hyiani A, Roff S. Perceptions of the educational environment of the medical school in King Abdul Aziz University. Med Teach 2004; 26 (6): 570-73.

12. Herrera C, Pacheco J, Rosso F, Cisterna C, Aichele D, Becker S, et al. Evaluación del ambiente educacional pre-clínico en seis Escuelas de Medicina en Chile. Rev Med Chile 2010; 138: 677-84.

13. Jakobsson U, Danielsen N, Edgren G. Psychometric evaluation of the Dundee Ready Educational Environment Measure: Swedish version. Med Teach 2011; 33: 267-74.

14. Rotthoff T, Ostapczuk M, De bruin J, Decking U, Schneider M, Ritz-timme S. Assesing the learning environment of a faculty: Psychometric validation of the German version of the Dundee Ready Education Environment Measure with students and teachers. Med Teach 2011; 33, 624-36.

15. Soares A, Almeida L, Diniz A, Almeida L. Construcción y validación de un modelo multidimensional de ajuste de los jóvenes al contexto universitario. Psicothema 2006; 18 (2): 249-55.

16. Soares A, Francischetto V, Dutra BM, Miranda J, Nogueira $\mathrm{C}$, Leme $\mathrm{V}$, et al. $\mathrm{O}$ impacto das expectativas na adaptaçao dos estudantes no Ensino Superior. Psico-USF 2014; 19 (1): 49-60. 
17. Meyer A, Ramírez L, Pérez C. Percepción de estrés en estudiantes chilenos de Medicina y Enfermería. Rev Educ Cienc Salud 2013; 10 (2): 79-85.

18. Sitticharoon C, Srisuma S, Kanavitoon S, Summachiwakij S. Exploratory study of factors related to educational scores of first preclinical year medical students. Adv Physiol Educ 2014; 38 (1): 25-33.

19. Petra I, Ramírez E, Flores A, Gutiérrez L, Granados C. (2002). Expectativas de formación médica de alumnos que ingresan al tercer año. Rev Fac Med UNAM 2002; 45 (5): 220-4.

20. Wouters A, Bakker AH, van Wijk IJ, Croiset G, Kusurkar RA. A qualitative analysis of statements on motivation of applicants for medical school. BMC Med Educ 2014; 14 (1): 200.

21. Rivera M, Milicic N. Alianza familia-escuela: percepciones, creencias, expectativas y aspiraciones de padres y profesores de enseñanza general básica. Psykhe 2006; 15 (1): 119-35.

22. García-Huidobro D, Núñez F, Vargas P, Astudillo S, Hitschfeld M, Gennero R, et al. Expectativas de estudiantes de medicina de pregrado en relación al perfil de médico esperado. Rev Med Chile 2006; 134 (8): 947-54.

23. van Roermund TA, Mokkink HG, Bottema BJ, van Weel C, Scherpbier AJ. Comparison of expectations and beliefs about good teaching in an academic day release medical education program: a qualitative study. BMC
Med Educ 2014; 14 (1): 211.

24. Elias M. Los abandonos universitarios: Retos ante el Espacio Europeo de Educación Superior. Estudios sobre Educación 2008; 15: 101-21.

25. Díaz C. Modelo conceptual para la deserción estudiantil universitaria chilena. Estud pedagóg 2008; 34 (2): 65-86.

26. Abello R, Díaz A, Pérez MV, Almeida LS, Lagos I, González J, et al. Vivencias e implicación académica en estudiantes universitarios: adaptación y validación de escalas para su evaluación. Estud pedagóg 2012; 38 (2): 7-19.

27. Martínez R, Hernández MJ, Hernández MV. Psicometría. Madrid: Alianza; 2006.

28. Tabachnick BG, Fidell LS. Using multivariate statistics. New York: Harper and Row; 2006.

29. Nunnally J, Bernstein I. Teoría psicométrica. México: Trillas; 1999.

30. Hair JF, Black WX, Babin BJ, Anderson RE, Tatham RL. Análisis multivariante. Madrid: Prentice Hall; 2005.

31. Costa AR, Araújo AM, Diniz AM, Almeida LS Expectativas académicas segundo o género dos estudantes portugueses do primeiro ano do ensino superior. International Journal of Developmental and Educational Psychology 2014; 1 (2): 325-34.

32. George D, Mallery P. SPSS for Windows step by step: A simple guide and reference. 11.0 update (4th ed.). Boston: Allyn \& Bacon; 2003. 